\title{
Optimal Pose Estimation Method for a Multi-Segment, Programmable Bevel-Tip Steerable Needle
}

\author{
Alberto Favaro*a ${ }^{*}$, Riccardo Secoli ${ }^{b}$, Ferdinando Rodriguez y Baena ${ }^{b}$, and Elena De Momi ${ }^{a}$
}

\begin{abstract}
Needle pose tracking is fundamental to achieve a precise and safe insertion in minimally-invasive percutaneous interventions. In this work, a method for estimating the full pose of steerable needles is presented, considering a foursegment Programmable Bevel-Tip Needle $(\mathrm{PBN})$ as a case study. The method estimates also the torsion of the needle that can arise during the insertion because of the interaction forces exerted between the needle and the insertion medium. A novel 3D kinematic model of the PBN is developed and used to predict the full needle pose during the insertion through an Extended Kalman Filter. The filter uses the position measurements provided by electromagnetic sensors located at the tip of the PBN segments as measurement data. The feasibility of the proposed solution is verified through ingelatin experiments, demonstrating remarkable performance with small errors in position (RMSE $<1 \mathrm{~mm}$ ) and orientation $\left(\mathrm{RMSE}<3^{\circ}\right)$ estimation, as well as good accuracy compared to a bespoke geometric pose reconstruction method.
\end{abstract}

\section{INTRODUCTION}

In minimally-invasive percutaneous interventions, the ability to finely control the needle during the insertion is fundamental to accurately reach the desired target location and follow the planned trajectory avoiding damages to the nearby anatomical structures. With steerable needles [1-4], factors like tissue inhomogeneity, soft tissues deformation, swelling and respiration can determine the needle deflection during the insertion [5]. Additionally, for some steerable needle designs, needle-tissue interaction forces have been shown to produce a torsional moment on the needle shaft that results in a torsion of the needle about its insertion axis. To compensate for unmodelled needle deflection and torsion, the control system of a steerable needle must know the position and the orientation (i.e. the full pose) of the tip at every time step, thus a needle tracking solution is required. Steerable needles can be tracked using imaging methods such as ultrasounds (US) [6] and X-Ray fluoroscopy [7], or via electromagnetic (EM) tracking [8]. Generally, these approaches cannot provide the full, 6 Degrees of Freedom (DoF) pose of the needle: its small dimensions in fact preclude the possibility to accommodate a $6 \mathrm{DoF}$ EM sensor or, for imaging solutions, to visualize the needle rotation about the insertion axis (the needle torsion).

\footnotetext{
*This project has received founding from the European Union's Horizon 2020 research and innovation program under grant agreement No 688279.

${ }^{a}$ NearLab, Department of Electronics, Information and Bioengineering Department (DEIB), Politecnico di Milano, 20133, Milan, Italy alberto.favaro@polimi.it, elena.demomiapolimi.it

${ }^{b}$ Mechatronics In Medicine Laboratory, Mechanical Engineering Department, Imperial College London, U.K. f.rodriguezeimperial.ac.uk
}

In the present paper, a method for estimating the full pose of a steerable needle is described. The solution implements an Extended Kalman Filter (EKF) based on the kinematic model of the needle. To simulate a typical needle insertion scenario where a conventional imaging method is used, only the needle tip position is considered as measurement data to estimate the full pose. The presented approach can be embedded in the needle control framework to inform the controller about the current pose of the needle and compensate for the onset of needle deflection and torsion. As a case study, in this work the complex needle design of the Programmable Bevel-tip Needle (PBN) is used. The PBN is a flexible needle under development within the EU EDEN2020* project [3] designed for percutaneous intervention in neurosurgery (drug infusion and in-situ optical diagnosis). It is manufactured with four axially-interlocked slender sections, robotically actuated at the needle base so that to slide over one another along the main needle direction. The needle tip changes according to the offset generated between adjacent sections and determines the steering direction and the degree of curvature [9].

The paper is organized as follows. In Section II, the state of the art of pose estimation for percutaneous steerable needles is presented. Section III describes a novel kinematic model of the PBN and the design of the EKF. In Section IV and Section V, the experimental setup and the results of the experiments performed in gelatin are reported. Sections VI and VII present the discussion and the overall conclusions of the work.

\section{RELATED WORKS}

In literature, a number of solutions have been formulated for steerable needle tracking. In the work of Vrooijink et al. [6], a US probe incrementally moves to track the position of the needle tip during the insertion. Considering the $x$ axis as the needle insertion direction, the pitch and yaw angles have been determined using geometric considerations while the roll angle associated to the needle torsion has been considered identical to the one measured at the needle base. This choice rely on the assumption of an infinite torsional stiffness of the needle, and has been used also in other needle tracking solutions [10-12]. Although this assumption is plausible for some needle designs, for others the material properties combined with the interaction forces experienced by the needle during the insertion can lead to a torsion of the needle shaft and consequently to a difference in the roll angle exhibited at the tip with respect to the one at the base. This is the case of the PBN, the kinematic model of which 
was proposed in 2D [13] and in 3D [14]. More recently, a dynamic model of the PBN based on the Euler-Bernoulli beam theory was presented [9]. Also these models, however, assume an infinite torsional stiffness of the needle, which was shown experimentally to not fit the real PBN behaviour for some needle configurations.

To perform a full, 6DoF pose estimation, Reed et al. [2] proposed a model-based Luenberger observer [1]. In their work, the $6 \mathrm{DoF}$ pose of the needle tip can be estimated using measurement data of needle tip position obtained from techniques such as biplane fluoroscopy or 3D US, and the torsional windup exhibited by the needle is corrected using the model of torsional dynamics presented in [15]. This approach has been successively extended by Swensen et al. [16] in a closed-loop framework, considering the torsional dynamics as depending upon the insertion length and obtaining relevant improvements in the control performance over purely kinematics control methods.

The measurement collected by a number of Fibre Bragg Grating (FBG) sensors embedded in four multi-core optical fibers are used in [17] to reconstruct the full pose of the needle, using the PBN as a case study. The possibility of reconstruction errors have been pointed out by the authors and attributed to a discrepancy in the level of torsion experienced by the optical fibers with respect to the needle shaft as they are not bounded together.

Optimal estimation algorithms have been used in the literature for needle tracking. In [18], Carriere et al. presented a method for the real-time shape prediction of a needle using a particle filter to address the uncertainty related to the noisy needle position measurements gathered from a transrectal US probe. An EKF combined with a deflection model has been used in [8] to merge the position information from two EM sensors located on the needle tip and the needle base and achieve a precise estimation of the tip position.

In our previous work [19], a method based on an EKF and a 2D kinematic model of the PBN was proposed for the pose tracking of a simplified, two-segment PBN. In this paper, the approach described in [19] is extended to the case of a foursegment PBN needle, for which a novel 3D kinematic model is developed. The full pose estimation is achieved using an EKF framework with the $3 \mathrm{DoF}$ position information of each PBN segment used as measurement data. The assumption of infinite needle stiffness is overtaken by including a torsion in the needle model. The performance of the filter has been tested through in-gelatin experiments.

\section{METHODS}

\section{A. The needle kinematic model}

In [13], Ko et al. proposed a 2D kinematic model for a two-segment PBN. The relative displacement between the two segments generates an offset at the tip that makes the needle steering on a plane. In the light of Ko's approach, a novel kinematic model for the four-segment PBN and spatial 3D steering is developed. This needle is viewed as the combination of two, two-segment needle systems, orthogonally positioned so that the first, $S_{H}$, controls the horizontal steering on the $x y$ plane and the second, $S_{V}$, the vertical steering on the $x z$ plane, as shown in Fig. 1a. The needle is oriented as shown in the bottom-left part of Fig. $1 \mathrm{~b}$, corresponding to the configuration in [14] twisted of $\pi / 4$ about the $x$ axis.

From [13], the kinematic models of the two systems can be written as:

$$
\begin{aligned}
& S_{H}: {\left[\begin{array}{c}
x_{H} \\
\dot{y_{H}} \\
\dot{\psi} \\
\dot{\delta_{H}}
\end{array}\right]=\left[\begin{array}{c}
\cos (\psi) \\
\sin (\psi) \\
k_{1}\left(\delta_{H}-\epsilon \psi\right) \\
0
\end{array}\right] v_{1}+\left[\begin{array}{l}
0 \\
0 \\
0 \\
1
\end{array}\right] v_{2, H} } \\
& S_{V}:\left[\begin{array}{c}
\dot{x_{V}} \\
\dot{z_{V}} \\
\dot{\theta} \\
\dot{\delta_{V}}
\end{array}\right]=\left[\begin{array}{c}
\cos (\theta) \\
-\sin (\theta) \\
k_{1}\left(\delta_{V}-\epsilon \theta\right) \\
0
\end{array}\right] v_{1}+\left[\begin{array}{l}
0 \\
0 \\
0 \\
1
\end{array}\right] v_{2, V}
\end{aligned}
$$

Where $x_{H}, y_{H}$ are the coordinates of the local frame $\mathbf{X}_{r, H}$ positioned on the tip of the rear segment of $S_{H}$. The angle $\psi$ is the steering angle about the $z$ axis and defines the curvature on the $x y$ plane. This angle is determined by the offset $\delta_{H}$ between the two segments measured at the needle base. The same considerations apply for $S_{V}$, with $x_{V}, z_{V}$ being the coordinates of the local frame $\mathbf{X}_{r, V}$ and $\theta$ the steering angle about the $y$ axis, determined by $\delta_{V}$. The coefficient $k_{1}$ is estimated in [13] and links the offset with the steering angle. The components $\epsilon \psi$ and $\epsilon \theta$ are included to correct the discrepancy caused by the needle steering between the offset at the needle base and the one at the tip, with $\epsilon$ function of the needle radius (see [13]). The velocities $v_{1}$ and $v_{2}$ represent the insertion speed of the needle during the insertion and the rate of change in $\delta_{H}$ and $\delta_{V}$. The values $\delta_{H}$ and $\delta_{V}$ define the radii of curvature $R_{H}$ and $R_{V}$ of the needle in the horizontal and vertical planes, i.e. two virtual planes that rotate together with the current configuration of the needle tip. The radii of curvature are expressed as:

$$
R_{H}=\frac{1}{k_{1}\left(\delta_{H}-\epsilon \psi\right)} \quad R_{V}=\frac{1}{k_{1}\left(\delta_{V}-\epsilon \theta\right)}
$$

The position and orientation of the leading segments, $\mathbf{X}_{l, H}$ and $\mathbf{X}_{l, V}$, with respect to $\mathbf{X}_{r, H}$ and $\mathbf{X}_{r, V}$ are defined as:

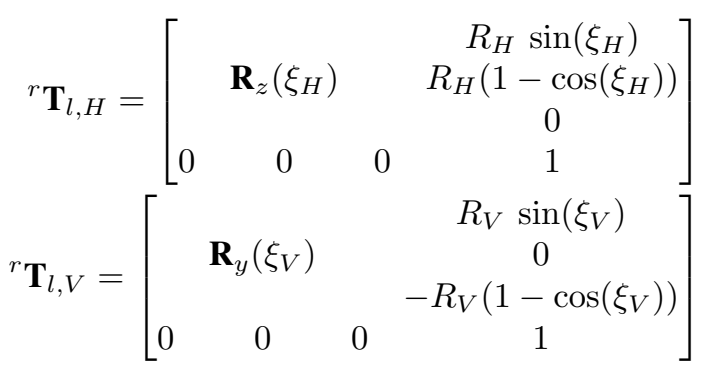

with $\mathbf{R}$ representing a rotation about the specified axis and $\xi_{V}, \xi_{H}$ the angles between $\mathbf{X}_{r, H}$ and $\mathbf{X}_{l, H}$ and between $\mathbf{X}_{r, V}$ and $\mathbf{X}_{l, V}$, respectively, as in Fig. 1a.

By merging the horizontal and vertical steering of $S_{H}$ and $S_{V}$, the four-segment PBN model is obtained, a schematic representation of which is reported in Fig. 1b. The resulting 
kinematic model of the PBN is:

$$
\left[\begin{array}{c}
\dot{x} \\
\dot{y} \\
\dot{z} \\
\dot{\theta} \\
\dot{\psi} \\
\dot{\delta}_{V} \\
\dot{\delta}_{H}
\end{array}\right]=\left[\begin{array}{c}
\cos (\theta) \cos (\psi) \\
\cos (\theta) \sin (\psi) \\
-\sin (\theta) \\
k_{1}\left(\delta_{V}-\epsilon \theta\right) \\
k_{1}\left(\delta_{H}-\epsilon \psi\right) \\
0 \\
0
\end{array}\right] v_{1}+\left[\begin{array}{cc}
0 & 0 \\
\vdots & \vdots \\
0 & 0 \\
1 & 0 \\
0 & 1
\end{array}\right] \mathbf{v}_{2}
$$

Similarly to (1), $x, y, z$, represent the coordinates of the rear segment $\mathbf{X}_{r}$ (in the case reported in Fig. $1 \mathrm{~b}, \mathbf{X}_{r}=\mathbf{X}_{1}$ ). The quantities $\theta$ and $\psi$ are the steering angles, while $\delta_{V}$ and $\delta_{H}$ are the offsets between segments 1 and 3 for the vertical displacement, and between segment 2 and 4 for the horizontal displacement. The correction factor $\epsilon$ is $\epsilon=$ $8 r_{n} \sqrt{2} /(3 \pi)$ for a four-segment needle, with $r_{n}$ the needle radius.

Said $l_{1}, l_{2}, l_{3}, l_{4}$ the insertion lengths of the four segments, the vector of the offset velocities is:

$$
\mathbf{v}_{2}=\left[\frac{d\left(1_{1}-l_{3}\right)}{d t}, \frac{d\left(1_{2}-l_{4}\right)}{d t}\right]^{T}
$$

and the insertion speed $v_{1}=\frac{d l_{\mathrm{r}}}{d t}$.

From (4), we define the pose of $\mathbf{X}_{r}$ as:

$$
{ }^{O} \mathbf{T}_{r}=\mathbf{P}(x, y, z) \mathbf{R}_{z}(\psi) \mathbf{R}_{y}(\theta)
$$

where $\mathbf{P}$ represents a pure translation.

We call $\delta_{t, i}$ the offset between the rear segment $\mathbf{X}_{r}$ and the tip $\mathbf{X}_{i}$ of segment $i, i \in 1, \ldots, 4$, as in Fig. 1b. The offset is defined as follows:

$$
\delta_{t_{i}}= \begin{cases}l_{i}-l_{r}-\epsilon \theta, & \text { if } i=1,3 \wedge i \neq r \\ l_{i}-l_{r}-\epsilon \psi, & \text { if } i=2,4 \wedge i \neq r \\ 0, & \text { if } i=r\end{cases}
$$

where the $\epsilon \theta$ and $\epsilon \psi$ are the offset corrections due to the needle steering. Any variation in the length of the segments due to compression is considered negligible.

As in (3), for each segment $i$ we define two angles $\left(\xi_{V_{i}}, \xi_{H_{i}}\right)$ between $\mathbf{X}_{r}$ and $\mathbf{X}_{i}$ in the vertical and horizontal axes as:

$$
\xi_{V_{i}}=\delta_{t_{i}} / R_{V} \quad \xi_{H_{i}}=\delta_{t_{i}} / R_{H}
$$

with $R_{V}$ and $R_{H}$ coming from (2). As in (3), the transformation ${ }^{r} \mathbf{T}_{i}$ between $\mathbf{X}_{r}$ and $\mathbf{X}_{i}$ is:

$$
{ }^{r} \mathbf{T}_{i}=\mathbf{P}\left(x_{r, i}, y_{r, i}, z_{r, i}\right) \mathbf{R}_{z}\left(\xi_{H_{i}}\right) \mathbf{R}_{y}\left(\xi_{V_{i}}\right)
$$

where the translation components are:

$$
\begin{aligned}
x_{r, i} & =\max \left(R_{H} \sin \left(\xi_{H_{i}}\right), R_{V} \sin \left(\xi_{V_{i}}\right)\right) \\
y_{r, i} & =R_{H}\left(1-\cos \left(\xi_{H_{i}}\right)\right) \\
z_{r, i} & =-R_{V}\left(1-\cos \left(\xi_{V_{i}}\right)\right)
\end{aligned}
$$

The transformation ${ }^{O} \mathbf{T}_{i}$ between $O$ and each $\mathbf{X}_{i}$ is:

$$
{ }^{O} \mathbf{T}_{i}={ }^{O} \mathbf{T}_{r}{ }^{r} \mathbf{T}_{i}
$$

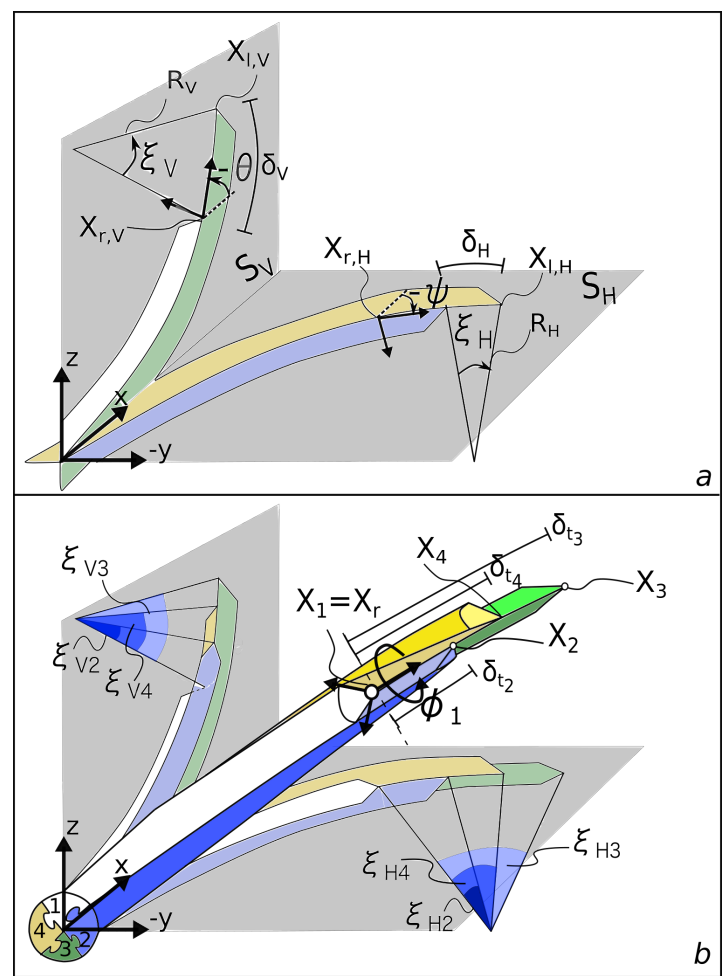

Fig. 1: In a), $S_{H}$ and $S_{V}$ are reported. The two segments composing $S_{H}$ are in blue and yellow, while the two composing $S_{V}$ are in green and white. The rear segments $\mathbf{X}_{r, V}$ and $\mathbf{X}_{r, H}$ as well as the leading segments $\mathbf{X}_{l, V}$ and $\mathbf{X}_{l, H}$ are shown. The two steering angles $(\psi, \theta)$ defined by $\delta_{H}$ and $\delta_{V}$ are plotted, together with the curvature radii and the angles $\xi_{V}$ and $\xi_{H}$. In b), the 3D representation of the four-segment PBN is shown. The frames $\mathbf{X}_{1, \ldots, 4}$ are reported. In this case, $\mathbf{X}_{r}=\mathbf{X}_{1}$ and $\phi_{1}$ is the torsion affecting segment 1 . The offsets $\delta_{t_{i}}$ at the level of the needle tip are reported (as $\left.\mathbf{X}_{r}=\mathbf{X}_{1}, \delta_{t_{1}}=0\right)$ ). The ideal 2D PBN projections are depicted on the $x z$ and $x y$ planes, which refer to the needle without torsion. The angles $\xi_{v_{i}}$ and $\xi_{h_{i}}$ can be identified in the figure.

\section{B. Needle torsion}

The material properties of the probe combined with the frictional and shear forces experienced in the interaction with the medium during the insertion determine a torsion of the needle along its shaft and a mismatch between the orientation shown at the needle base and the one at the tip. The torsion consists in a rotation by an angle $\phi$ about the $x$ axis (see Fig. 1b). In the case of the PBN, to make the needle steering in the space, an offset is generated among the four needle segments. This turns in a different insertion length for each needle segment, which is supposed to be associated to a different level of torsion (i.e. the leading segments is expected to show a larger torsion than the rear segment). In the light of this, a torsion angle $\phi_{i}$ is associated to each needle segment $i$, whose tip pose becomes:

$$
{ }^{O} \mathbf{T}_{i}^{\prime}=\mathbf{R}_{x}\left(\phi_{i}\right)^{O} \mathbf{T}_{i}
$$

\section{Needle pose estimation}

Hereinafter, the EKF is presented. The state vector $\mathbf{x}$ describes the needle status and is defined as follows:

$$
\mathbf{x}=\left[x, y, z, \theta, \psi, \phi_{1}, \phi_{2}, \phi_{3}, \phi_{4}, \delta_{v}, \delta_{h}, \delta_{t_{1}}, \delta_{t_{2}}, \delta_{t_{3}}, \delta_{t_{4}}\right]^{T}
$$


where the first 5 parameters come from (4), $\delta_{t_{1, \ldots 4}}$ from (7) and $\phi_{1, \ldots, 4}$ express the torsion experienced by the four segments. The state at time $k$ evolves according to:

$$
\mathbf{x}(k+1)=f(\mathbf{x}(k))+b(\mathbf{u}(k+1))+n(k+1)
$$

where $\mathbf{u}$ is the vector of inputs, represented by $v_{1}$ and the segment insertion lengths:

$$
\mathbf{u}=\left[v_{1}, l_{1}, l_{2}, l_{3}, l_{4}, l_{r}\right]^{T}
$$

where $l_{r}=\min \left(l_{1}, l_{2}, l_{3}, l_{4}\right)$.

The process noise $n(k)$ is assumed to be drawn from a zero-mean Gaussian distribution $n(k) \sim \mathcal{N}\left(0, \mathbf{Q}_{p}\right)$ with variance $\mathbf{Q}_{p}$, as in [20]. The value of $\mathbf{Q}_{p}$ is set empirically in the filter tuning.

The update function $f(\cdot)$ is defined as:

$$
\begin{aligned}
f(\mathbf{x}(k))= & {\left[x(k), y(k), z(k), \theta(k), \psi(k), \phi_{1}(k), \phi_{2}(k), \phi_{3}(k),\right.} \\
& \left.\phi_{4}(k), 0,0,-\epsilon \theta(k),-\epsilon \psi(k),-\epsilon \theta(k),-\epsilon \psi(k)\right]^{T}
\end{aligned}
$$

The control-input function $b(\cdot)$ is defined as:

$$
b(\mathbf{u}(k+1))=\left[\begin{array}{c}
\cos (\theta(k)) \cos (\psi(k)) \Delta t \cdot v_{1}(k+1) \\
\cos (\theta(k)) \sin (\psi(k)) \Delta t \cdot v_{1}(k+1) \\
-\sin (\theta(k)) \Delta t \cdot v_{1}(k+1) \\
k_{1}\left(\delta_{v}(k)-\epsilon \theta(k)\right) \Delta t \cdot v_{1}(k+1) \\
k_{1}\left(\delta_{h}(k)-\epsilon \psi(k)\right) \Delta t \cdot v_{1}(k+1) \\
0 \\
\vdots \\
0 \\
l_{1}(k+1)-l_{3}(k+1) \\
l_{2}(k+1)-l_{4}(k+1) \\
l_{1}(k+1)-l_{r}(k+1) \\
l_{2}(k+1)-l_{r}(k+1) \\
l_{3}(k+1)-l_{r}(k+1) \\
l_{4}(k+1)-l_{r}(k+1)
\end{array}\right]
$$

where $\Delta t$ is the sampling time.

The measurement data can be retrieved by embedded sensors, e.g. electromagnetic sensors, or via a suitable imaging modality. The observation at time $k$ is expressed as:

$$
y(k)=h(\mathbf{x}(k))+v(k)
$$

where the measurement function $h(\cdot)$ is the results of a nonlinear function defined as:

$$
h(\mathbf{x}(t))=\left[\mathbf{p}_{1}, \mathbf{p}_{2}, \mathbf{p}_{3}, \mathbf{p}_{4}\right]^{T}
$$

with $\mathbf{p}_{i}$ is the translation component of ${ }^{O} \mathbf{T}_{i}^{\prime}$ from (12), representing the $x, y, z$ coordinates of the tip of each needle segment. The measurement noise, $v(k)$, represents the noise over the EM measurement, for which a zero-mean Gaussian distribution is supposed, as in [20], i.e. $v(k) \sim \mathcal{N}\left(0, \mathbf{Q}_{m}\right)$. Variance $\mathbf{Q}_{m}$ is unknown a-priori and has to be tuned in the filter calibration on the basis of the experimental measurement data.

\section{EXPERIMENTAL PROTOCOL}

A total of three needle insertions were performed in gelatin. For each insertion, a different configuration of the needle segments was generated to perform a different needle path. As in previous evaluation studies (e.g. [9]), a 10\% by weight bovine gelatin (Chef William Powdered Gelatin) was used, the shear modulus of which $(7.72 \pm 0.08 \mathrm{kPa})$ can be considered an acceptable approximation for human brain white matter [9]. The insertions were performed using a medical-grade, $2.5 \mathrm{~mm}$ outer diameter PBN, the detailed description of which can be found in [9]. The PBN has $0.3 \mathrm{~mm}$ diameter lumens tunnelled in each segment. These lumens were used to accommodate four EM Aurora ${ }^{\mathrm{TM}}$ sensors (5DoF, 0.3mm diameter, Northern Digital inc.). In this work, only the $3 \mathrm{DoF}$ sensors positions have been used as measurement data to guarantee the applicability of the method in conditions where the orientation cannot be measured directly, as is the case for many practical implementations where standard imaging modalities are used.

In the tests, the estimated needle position was compared with the EM measurement in terms of RMSE to verify the filter accuracy. Orientation measurements (pitch and yaw angles) from the EM sensors are used as ground truth to validate the EKF estimation performance. A geometric approach is exploited to have a term of comparison for the roll angle estimated by the EKF. For each time step, the geometric method defines a plane orthogonal to the insertion axis. Then, a centerline point is defined by averaging the position of the four points corresponding to the intersection of the EM measurement traces with the orthogonal plane. The local frame is centered on the centerline, having as $x$ axis the needle insertion and as $y$ and $z$ axes the directions from the centerline to the intersection points between the traces of sensor 4 and sensor 1 with the orthogonal plane. One needle insertion is used to evaluate the benefits provided by the EKF framework with respect to the use of the needle kinematic model alone. The same insertion is also used to assess the robustness of the EKF by adding further noise (Gaussian noise, $\mathcal{N}\left(0,1 \mathrm{~mm}^{2}\right)$ ) to the measurement data; in this case, position and orientation estimation errors are computed considering the original EM measurements.

The measurement noise variance $\left(Q_{m}\right)$ was chosen empirically by tuning the nominal accuracy of the EM sensors to the value that guarantees the best estimation performance, i.e. $Q_{m}=1 \mathrm{~mm}^{2}$. Similarly, the process variance $\left(Q_{p}\right)$ was chosen as $Q_{p}=1 \cdot 10^{-2}$ as the value that provides the best prediction accuracy.

The experimental setup is reported in Fig. 2 and described hereinafter. A robotic system featuring four linear actuators (one per segment, linear pitch $1 \mathrm{~mm} / \mathrm{rev}$ ) drives the needle during the insertion. Per each segment, a rotational encoder at the motor (Maxon motor DC16XS - Res 1200 pulse/rev) assures the precision motion of each segment and records the insertion length from which the parameters $v_{1}$ and $\mathbf{v}_{2}$ can be computed. The needle is inserted for $20 \mathrm{~mm}$ in the gelatin with all four segments aligned to distance the 


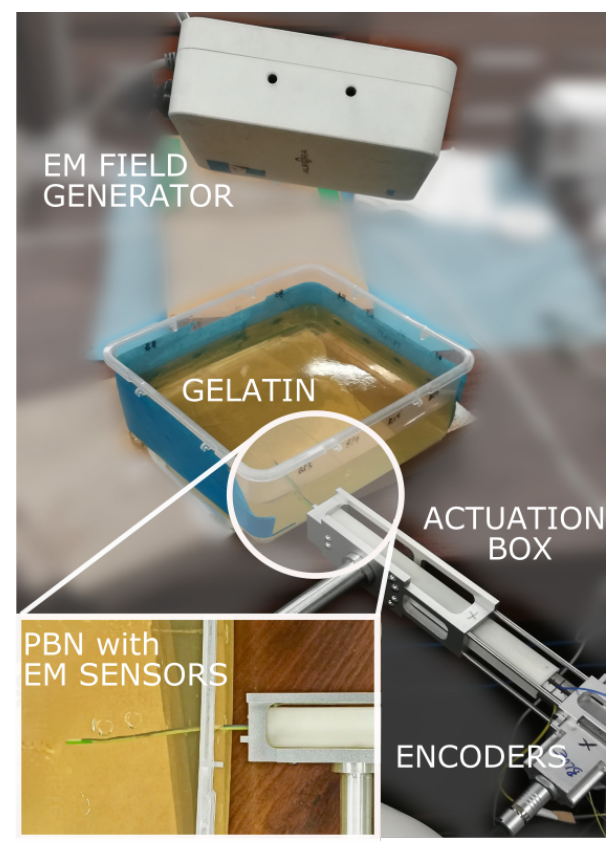

Fig. 2: The experimental setup (from [19]). The PBN is inserted in the gelatin. The EM field generator is located on top of the gelatin box for tracking the four EM sensors embedded in each segment of the PBN. An actuation box composed by four linear motors and rotational encoders controls the insertion of each needle segment and measures the insertion length. A magnification of the needle insertion is shown on the bottom left.

EM sensors from the trocar tube and thus reduce possible magnetic disturbances. Then, the offset among the segments is generated and the needle is inserted at a constant speed of $1 \mathrm{~mm} \cdot \mathrm{sec}^{-1}$. A sample rate of $2 \mathrm{~Hz}$ is used, which was assumed as appropriate considering the low insertion speed. A desktop PC with Linux Ubuntu 16.04 operating system running the Robotic Operating System (ROS) is used to control the needle insertion and for data storage.

\section{RESULTS}

In Tab. I, the performance of the needle kinematic model with and without the EKF are reported in terms of Root Mean Square Error (RMSE) in position and orientation for insertion 1, together with the estimation RMSE of the EKF in the case of additional noise on the measurement data. The roll angle is omitted as the model cannot compute it by itself only on the basis of the model inputs.

The results from the three insertions in gelatin are reported in the graphs of Fig. 3. In the left side, the original position measurements from the four EM sensors are reported along with the reconstruction of the needle obtained from the EKF position and orientation estimation (the CAD model used as a reconstruction of the needle shaft is shown with its local reference frames in the top-left corner of Fig. 3). In the right side of Fig. 3, the graphs report the segments tip position and orientation for each insertion over time. A comparison between the measured and estimated position is possible by looking at the graphs of the $x-y-z$ coordinates (for readability, EM measurements are shown under-sampled by a factor 20). Orientation angles $(\phi, \theta, \psi)$ are also reported. For the pitch and yaw angles, the orientation measured by the EM sensors are reported as terms of comparison (the roll angle cannot be measured as we used 5DoF sensors). In insertion 1 and 2, the segments orientation estimated by the EKF can be compared also to the results from the geometrical method. In insertion 3, the geometric pose computation was not possible due to the impossibility to define the needle centerline when the four segments are not completely paired side by side.

TABLE I: Comparison in terms of RMSE in position and orientation for insertion 1. The needle kinematic model is tested alone and in combination with the EKF, which is also assessed in terms of robustness in case of additional noise added on the measurement data. The roll angle is not included as no estimation is possible for this quantity using the model alone.

\begin{tabular}{cccccc}
\hline & \multicolumn{3}{c}{ Position error $[\mathrm{mm}]$} & \multicolumn{2}{c}{ Orientation error [deg] } \\
\hline & $x$ & $y$ & $z$ & $\theta$ & $\psi$ \\
\hline no EKF & 1.44 & 1.53 & 1.69 & 2.95 & 3.43 \\
EKF & 0.9 & 0.13 & 0.16 & 1.28 & 1.39 \\
EKF + noise & 0.9 & 0.21 & 0.24 & 1.61 & 1.29
\end{tabular}

\section{DISCUSSIONS}

The importance of including the needle kinematic model in an EKF framework is evidenced by the results reported in Tab. I, where the benefits in terms of position and orientation errors for the EKF are evident compared to the needle model alone. In the case of additional measurement noise, no significant variation is shown in the position and orientation errors. This proves the goodness of the needle model used, as well as the fact that the EKF does not over-rely on the EM measurements.

From the three insertions in gelatin, the ability of the filter to follow the position tracked by the EM sensors and estimate the full pose of each segment of the PBN is demonstrated.

The filter has a fast convergence in position tracking for all segments and in all the three insertions. The RMSE calculated over the $x, y, z$ position between the estimated segment tips position and the one measured by the EM sensors results $R M S E_{x y z}=(0.57 \mathrm{~mm}, 0.04 \mathrm{~mm}, 0.07 \mathrm{~mm})$. With regard to the orientation, a fast convergence can be noticed for the pitch $(\theta)$ and yaw $(\psi)$ angles. The accuracy of the pitch and yaw angle estimation can be appreciated by comparing the data with the ones measured by the EM sensors, resulting in a $R M S E_{\theta}=2.28^{\circ}$ and a $R M S E_{\phi}=$ $1.65^{\circ}$. The trends followed by the roll angles $(\phi)$ are less easy to evaluate as longer time for convergence is needed and no reference is available from the 5DoF EM sensors. In insertion 1 and 2, the comparison with the geometric approach suggests a correct estimation of the roll angle, but a quantitative one-to-one comparison for each specific needle segment is not possible as the geometric approach computes a unique frame on the needle centerline and thus no RMSE can be computed for the $\phi$ angle. In insertion 3, the fact that the segments are never fully paired makes impossible to use the geometric pose computation method. Still, despite 


\section{Insertion 1}
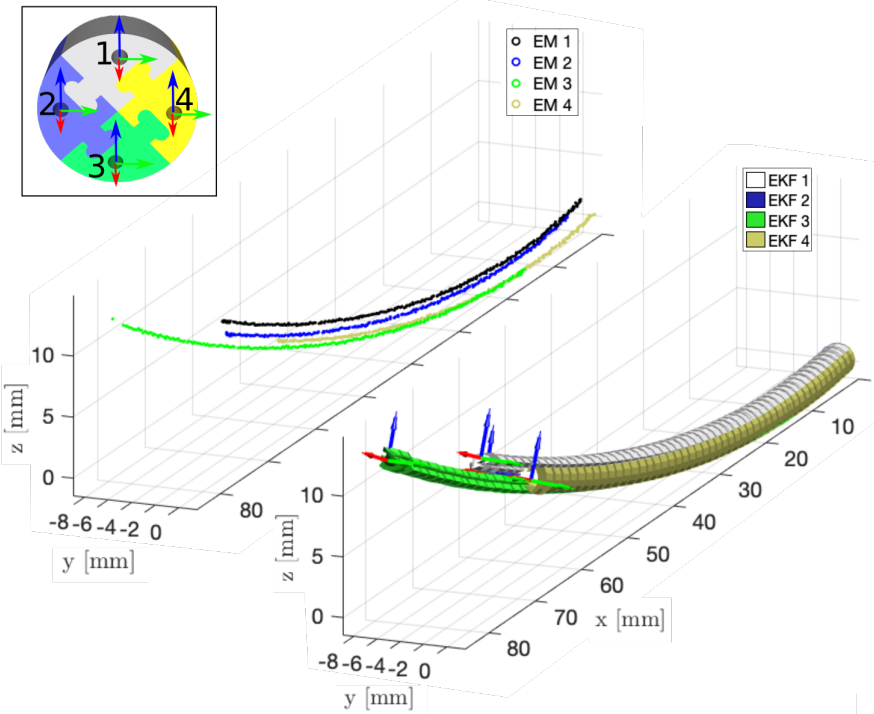

Insertion 2
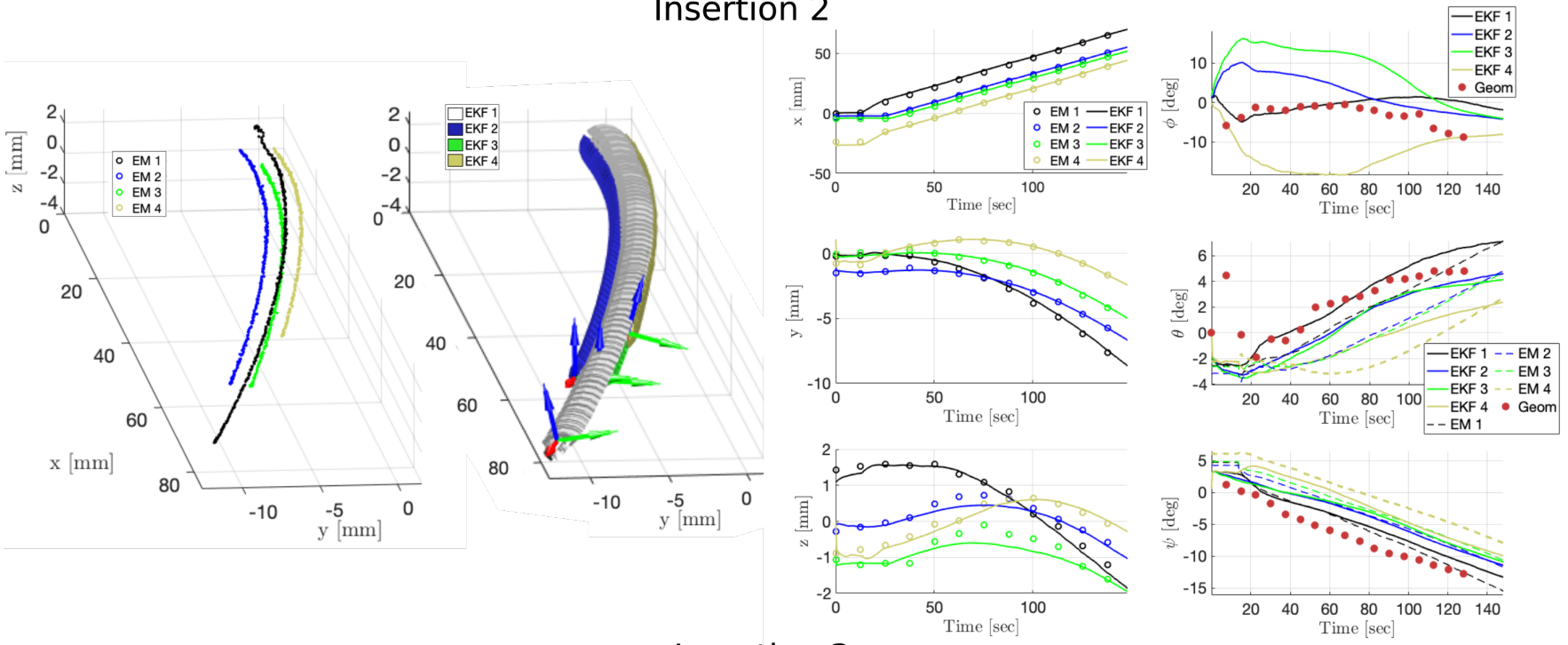

Insertion 3
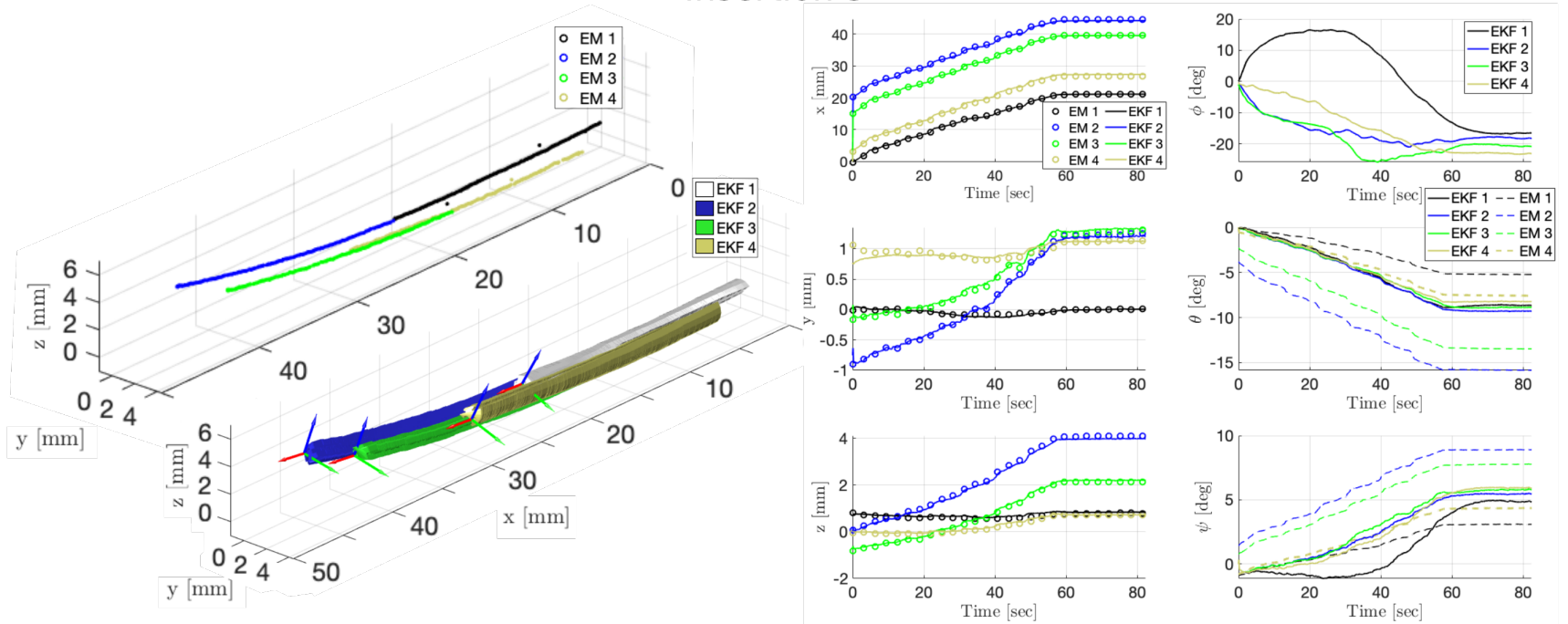

Fig. 3: The results from three PBN insertions in gelatin are reported. In the left part, the raw position measurements from the four EM sensors and the reconstructions of the needle obtained from the position and orientation estimated by the EKF are reported. In the top-left corner, the CAD model used for the reconstruction is shown. On the right, the graphs report the estimated position and orientation (EKF) for each insertion along with the measurement data (EM) and the orientation resulting from the geometric pose computation (Geom). In insertion 3 , the segments are never fully paired and this makes impossible to apply the geometric pose computation method. 
the initial rise, at the end of the insertion, the roll angle of segment 1 decreases to values similar to the ones of segment 2 and 3 at the start of the insertion. This suggests that, for the same insertion depth, the segments show the same level of torsion.

The comparison among the roll angles exhibited in the three different insertions is not straightforward. In insertion 1 , the PBN performs a deeper insertion in the gelatin with respect to insertion 3 , also achieving a larger steering, as evidenced by the higher displacement in the Cartesian axes. Nonetheless, the modulus of the roll angles exhibited by the PBN segments at the end of insertion 1 is considerably smaller than the one of insertion 3. This finds explanation in the different configurations featured by the needle, that determines a larger or a smaller torsion effect on the needle shaft according to each specific case: in insertion 1, the helical shape featured by the PBN may promote larger torsion compared to other needle configurations. This evidence, along with the magnitude of the roll angles estimated experimentally, validates the importance of a solution able to track the full pose of the needle during the insertion.

\section{CONCLUSION}

Tracking the tip of a steerable needle is crucial for enabling a proper control during the insertion in minimallyinvasive percutaneous interventions. The solution herein presented overtakes the assumption of an infinite torsional stiffness of steerable needles and proposes a method for the estimation of their full pose using the PBN as a case study. An EKF is used as an optimal estimator of the needle pose to correct the prediction from a novel $3 \mathrm{D}$ needle kinematic model through the position measurements gathered from four EM sensors located at the tip of the PBN segments (or from a suitable imaging modality). The solution was tested in brain-like gelatin on insertions with different needle configurations to assess its performance and stability. The results demonstrated the benefits and the feasibility of the proposed method, with low errors in position (RMSE $<1 \mathrm{~mm}$ ) and orientation ( $\mathrm{RMSE}<3^{\circ}$ ) when compared to the measurements from the EM sensors and the pose estimated by a bespoke geometric approach, even though long time of convergence was evidenced for the torsion angle.

Future studies will evaluate the performance of the presented solution using different PBN models, as the one in [14]. Additionally, the benefits derived from including the pitch and yaw angles measured by the 5DoF EM sensors in the EKF pose estimation will be investigated, together with possible enhancements related to the use of other optimal estimators such as the Unscented Kalman Filter or the particle filter.

\section{REFERENCES}

[1] V. Kallem and N. J. Cowan, "Image guidance of flexible tip-steerable needles," IEEE Transactions on Robotics, vol. 25, no. 1, pp. 191-196, 22009.

[2] K. B. Reed, A. Majewicz, V. Kallem, R. Alterovitz, K. Goldberg, N. J. Cowan, and A. M. Okamura, "Robot-assisted needle steering," IEEE Robotics and Automation Magazine, vol. 18, no. 4, pp. 35-46, 122011.
[3] R. Secoli and F. R. Y. Baena, "Adaptive path-following control for bio-inspired steerable needles," in Proceedings of the IEEE RAS and EMBS International Conference on Biomedical Robotics and Biomechatronics, vol. 2016-July. IEEE, 6 2016, pp. 87-93.

[4] N. J. van de Berg, D. J. van Gerwen, J. Dankelman, and J. J. van den Dobbelsteen, "Design Choices in Needle Steering-A Review," IEEE/ASME Transactions on Mechatronics, vol. 20, no. 5, pp. 2172-2183, 102015.

[5] S. Misra, K. B. Reed, B. W. Schafer, K. T. Ramesh, and A. M. Okamura, "Mechanics of flexible needles robotically steered through soft tissue," International Journal of Robotics Research, vol. 29, no. 13, pp. 1640-1660, 112010.

[6] G. J. Vrooijink, M. Abayazid, and S. Misra, "Real-time threedimensional flexible needle tracking using two-dimensional ultrasound," in Proceedings - IEEE International Conference on Robotics and Automation. IEEE, 5 2013, pp. 1688-1693.

[7] K. Ralovich, M. John, E. Camus, N. Navab, and T. Heimann, " $6 \mathrm{DoF}$ catheter detection, application to intracardiac echocardiography," Medical image computing and computer-assisted intervention : MICCAI ... International Conference on Medical Image Computing and Computer-Assisted Intervention, vol. 17, no. Pt 2, pp. 635-642, 2014.

[8] H. Sadjadi, K. Hashtrudi-Zaad, and G. Fichtinger, "Needle deflection estimation using fusion of electromagnetic trackers," in 2012 Annual International Conference of the IEEE Engineering in Medicine and Biology Society. IEEE, 8 2012, pp. 952-955.

[9] T. Watts, R. Secoli, and F. R. Y. Baena, "A Mechanics-Based Model for 3-D Steering of Programmable Bevel-Tip Needles," IEEE Transactions on Robotics, vol. 35, no. 2, pp. 371-386, 42019.

[10] N. Shahriari, E. Hekman, M. Oudkerk, and S. Misra, "Design and evaluation of a computed tomography (CT)-compatible needle insertion device using an electromagnetic tracking system and CT images," International Journal of Computer Assisted Radiology and Surgery, vol. 10, no. 11, pp. 1845-1852, 112015.

[11] M. Abayazid, M. Kemp, and S. Misra, "3D flexible needle steering in soft-tissue phantoms using Fiber Bragg Grating sensors," in Proceedings - IEEE International Conference on Robotics and Automation. IEEE, 5 2013, pp. 5843-5849.

[12] M. Abayazid, P. Moreira, N. Shahriari, S. Patil, R. Alterovitz, and S. Misra, "Ultrasound-guided three-dimensional needle steering in biological tissue with curved surfaces," Medical Engineering and Physics, vol. 37, no. 1, pp. 145-150, 12015.

[13] S. Y. Ko, L. Frasson, and F. Rodriguez Y Baena, "Closed-loop planar motion control of a steerable probe with a programmable bevel inspired by nature," IEEE Transactions on Robotics, vol. 27, no. 5, pp. 970-983, 102011.

[14] R. Secoli and F. Rodriguez Y Baena, "Closed-loop 3D motion modeling and control of a steerable needle for soft tissue surgery," in Proceedings - IEEE International Conference on Robotics and Automation. IEEE, 2013, pp. 5831-5836.

[15] K. Reed, A. Okamura, and N. Cowan, "Modeling and Control of Needles With Torsional Friction," IEEE Transactions on Biomedical Engineering, vol. 56, no. 12, pp. 2905-2916, 122009.

[16] J. P. Swensen, M. Lin, A. M. Okamura, and N. J. Cowan, "Torsional dynamics of steerable needles: Modeling and fluoroscopic guidance," IEEE Transactions on Biomedical Engineering, vol. 61, no. 11, pp. 2707-2717, 112014.

[17] F. Khan, A. Denasi, D. Barrera, J. Madrigal, S. Sales, and S. Misra, "Multi-Core Optical Fibers With Bragg Gratings as Shape Sensor for Flexible Medical Instruments," IEEE Sensors Journal, vol. 19, no. 14, pp. 5878-5884, 72019.

[18] J. Carriere, C. Rossa, R. Sloboda, N. Usmani, and M. Tavakoli, "Realtime needle shape prediction in soft-tissue based on image segmentation and particle filtering," in 2016 IEEE International Conference on Advanced Intelligent Mechatronics (AIM). IEEE, 7 2016, pp. 1204-1209.

[19] A. Favaro, R. Secoli, F. Rodriguez y Baena, and E. De Momi, "Modelbased robust pose estimation for a multi-segment,programmable beveltip steerable needle," IEEE Robotics and Automation Letters.

[20] S. Navaei Lavasani, M. Deevband, P. Farnia, A. Ahmadian, and S. Saghatchi, "Compensation of dynamic electromagnetic field distortion using simultaneous localization and mapping method with application in endobronchial ultrasound-transbronchial needle aspiration (EBUS-TBNA) guidance," International Journal of Medical Robotics and Computer Assisted Surgery, 2020. 